FRI0470

EFFECT OF DISCONTINUATION OF DENOSUMAB IN SUBJECTS WITH RHEUMATOID ARTHRITIS TREATED WITH GLUCOCORTICOIDS

Kenneth Saag ${ }^{1}$, Michele Mcdermott ${ }^{2}$, Jonathan Adachi $^{3}$, Willem Lems ${ }^{4}$, Nancy Lane ${ }^{5}$, Piet Geusens ${ }^{6}$, Peter Butler ${ }^{2}$, LI Chen ${ }^{2}$, Daria B. Crittenden ${ }^{2}$, Robin Dore ${ }^{7}$, Stanley Cohen $8 .{ }^{1}$ University of Alabama, Birminghanm, United States of America; ${ }^{2}$ Amgen Inc., Thousand Oaks, United States of America; ${ }^{3}$ McMaster University, Hamilton, Canada; ${ }^{4}$ VU University Medical Centre, Amsterdam, Netherlands; ${ }^{5}$ University of California at Davis Medical Center, Sacramento, United States of America; ${ }^{6}$ Maastricht University Medical Center, Maastricht, Netherlands; ${ }^{7}$ Robin K Dore Inc, Tustin, United States of America; ${ }^{8}$ Metroplex Clinical Research Center, Dallas, United States of America

Background: Denosumab, a monoclonal antibody against RANKL, is approved for the treatment of glucocorticoid (GC) induced osteoporosis (GiOP). In postmenopausal women with osteoporosis, denosumab discontinuation leads to a transient increase in bone turnover above baseline, peaking at 12 months from the last dose, and a corresponding decline in bone mineral density (BMD). To better understand the effects of denosumab discontinuation in GC-treated patients, we analyzed a subgroup receiving GCs at baseline from a phase 2 study of denosumab in subjects with rheumatoid arthritis (RA), followed for 12 months after denosumab discontinuation.

Objectives: To evaluate changes in bone turnover and BMD in subjects with RA on GCs treated with denosumab, after discontinuing denosumab for 12 months.

Methods: This double-blind, placebo-controlled study enrolled subjects with RA who were randomized to receive denosumab $60 \mathrm{mg}$, denosumab 180 $\mathrm{mg}$, or placebo subcutaneously for 12 months, and followed for progression of structural damage. Subjects were followed for an additional 12 months after denosumab discontinuation. Outcome measures in this subgroup analysis of subjects treated with GCs at study baseline included percent change from baseline in serum C-terminal telopeptide of type I collagen (CTX) and lumbar spine (LS) and total hip (TH) BMD on- and off-treatment. Baseline mean (SD) prednisone equivalent dose (mg/day) was 6.1 (2.4), 5.2 (2.1), and 6.1 (3.2) in the placebo, denosumab 60 $\mathrm{mg}$, and denosumab $180 \mathrm{mg}$ groups, respectively. Data on CTX are reported as median and interquartile range. Percent changes in LS and TH BMD at each time point were assessed based on a repeated-measures model adjusting for treatment, baseline use of steroids, previous use of biologics, and baseline BMD value.

Results: Among 218 subjects in the phase 2 study, 82 (26 placebo, 27 denosumab $60 \mathrm{mg}$, and 29 denosumab $180 \mathrm{mg}$ ) were included in this analysis. After 12 months of denosumab treatment, CTX decreased from baseline in both groups (Figure); in the off-treatment period, CTX returned to baseline by 18 months and was overall similar to placebo at 24 months. BMD increased at the LS and TH at 12 months with denosumab treatment (Figure) and returned to baseline levels after 12 months of discontinuation.

Conclusion: Like all non-bisphosphonate medications for osteoporosis, denosumab is reversible with discontinuation. In this small subgroup of GC-treated subjects with RA, BMD gains achieved with denosumab were lost upon discontinuation, consistent with observations in postmenopausal women receiving denosumab for osteoporosis. In this analysis of shortterm denosumab use in subjects with RA receiving GCs, bone turnover was reduced with denosumab and gradually returned to baseline upon discontinuation, without a clear increase to above-baseline levels in the off-treatment period.

Disclosure of Interests: Kenneth Saag Grant/research support from: Amgen, Ironwood/AstraZeneca, Horizon, SOBI, Takeda, Consultant for: Abbvie, Amgen, Ironwood/AstraZeneca, Bayer, Gilead, Horizon, Kowa, Radius, Roche/Genentech, SOBI, Takeda, Teijin, Michele McDermott Shareholder of: Amgen Inc., Employee of: Amgen Inc., Jonathan Adachi Grant/research support from: Amgen Inc, Consultant for: Amgen Inc, Eli Lilly, Speakers bureau: Amgen Inc., Willem Lems Speakers bureau: Amgen Inc., Merck, Eli Lilly and Pfizer, Nancy Lane Consultant for: Samumed, LLC, Piet Geusens Grant/research support from: Research support, consultant and/or speaker fees from: Pfizer, Abbott, Eli Lilly, Amgen, MSD, Roche, UCB, BMS, Novartis, Will-Pharma, Grant/research support from: Pfizer, Abbott, Lilly, Amgen, MSD, Will, Bio Minerals and Roche, Consultant for: Research support, consultant and/or speaker fees from: Pfizer, Abbott, Eli Lilly, Amgen, MSD, Roche, UCB, BMS, Novartis, Will-Pharma, Speakers bureau: Research support, consultant and/or speaker fees from: Pfizer, Abbott, Eli Lilly, Amgen, MSD, Roche, UCB, BMS, Novartis, Will-Pharma, Speakers bureau: Pfizer, Abbott, Lilly, Amgen, MSD, Will, Bio Minerals and Roche, Peter Butler Shareholder of: Amgen Inc., Employee of: Amgen Inc., Li Chen Shareholder of: Amgen Inc., Employee of: Amgen Inc., Daria B Crittenden Shareholder of: Amgen
Inc., Employee of: Amgen Inc., Robin Dore Grant/research support from: Amgen Inc., AbbVie, Biogen, Gilead, Pfizer, Eli Lilly, Novartis, and UCB, Consultant for: Amgen Inc., AbbVie, Gilead, Pfizer, Eli Lilly, Novartis, and UCB, Speakers bureau: Amgen Inc., AbbVie, Pfizer, BMS, Eli Lilly, Novartis, UCB, and Radius, Stanley Cohen Grant/research support from: AbbVie, Amgen Inc., AstraZeneca, Biogen-IDEC, Bristol Meyer Squibb, Genentech, Janssen, Eli Lilly, Novartis, Pfizer, Merck, and Roche, Consultant for: Abbvie, Amgen, AstraZeneca, Biogen-IDEC, Bristol Meyer Squibb, Genentech, Janssen, Lilly, Novartis Pfizer, Merck and Roche DOI: 10.1136/annrheumdis-2019-eular.1873

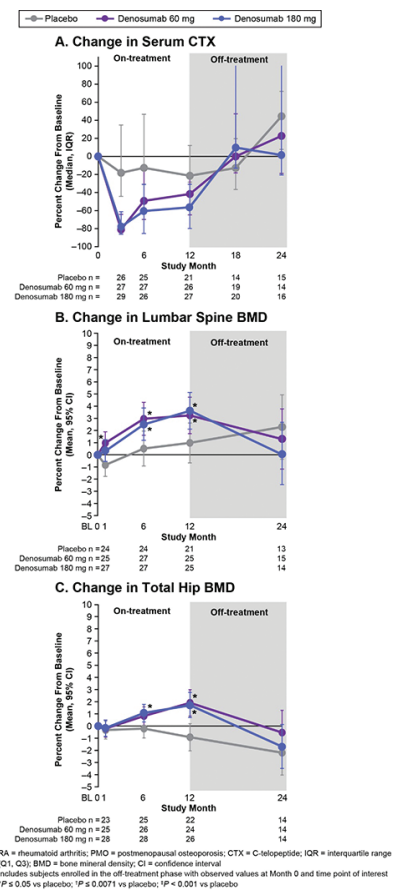

\section{FRI0471 BONE QUALITY ASSESSMENT IN PATIENTS WITH TYPE 2 DIABETES MELLITUS}

Nassima Hanouchine ${ }^{1,1}$, Salah Ouhadj ${ }^{2}$, Chafia Dahou-Makhloufi ${ }^{3} .{ }^{1} \mathrm{CHU}$ TIZI OUZOU, TIZI OUZOU, Algeria; ${ }^{2} E P H$ DELLYS, BOUMERDES, Algeria; ${ }^{3} \mathrm{CHU}$ BABELOUED, ALGIERS, Algeria

Background: Type 2 diabetes mellitus (T2DM) is a risk factor for osteoporotic fractures although bone mineral density is normal or even increased Thus, diabetes may be associated with a reduction of bone strength that is not reflected in the measurement of bone mineral density (BMD)

Objectives: The aim of this study was to compare BMD with a non invasive assessment of trabecular microarchitecture, (Trabecular bone score) TBS, in patients with T2DM

Methods: In a prospective cross-sectional study, trabecular microarchitecture was examined in patients with T2DM and non diabetic control subjects. The exclusion criteria were diseases (hyperthyroidism, Cushing's syndrome, primary hyperparathyroidism, renal failure, malabsorption), rheumatic diseases and/or medications that might affect bone and mineral metabolism,post menopausal women. Lumbar spine BMD was measured by dual-emission $x$-ray absorptiometry (DXA), and TBS was calculated by examining pixel variations within the DXA images using TBS iNsight

Results: 205 patients (108 male, 97 female), aged 25 to 60 years with T2DM and 205 non diabetic control subjects ((105 male, 100 female) Mean TBS was lower in T2DM $(1.256 \pm 0.103$ vs. $1.291 \pm 0.101, p=0.001)$ Mean BMD was higher in T2DM $Z$ score $(0.516 \pm 1.346$ vs. -0.8151 $\pm 1.153, p=0.016$ )

Conclusion: In T2DM,TBS is lower than control subjects. Abnormal trabecular microarchitecture may help explain the paradox of increased fractures at a higher BMD in T2DM

\section{REFERENCES :}

[1] Dhaliwal R, Cibula D, Ghosh C, Weinstock RS, Moses AM. Bone qualityassessment in type 2 diabetesmellitus. OsteoporosInt.2014;25:1969-73. - 